\title{
Can a periodically driven particle resist laser cooling and noise?
}

\author{
A. Maitra, ${ }^{1}$ D. Leibfried, ${ }^{2}$ D. Ullmo, ${ }^{1}$ and H. Landa ${ }^{3, *}$ \\ ${ }^{1}$ LPTMS, CNRS, Université Paris-Saclay, 91405 Orsay, France \\ ${ }^{2}$ National Institute of Standards and Technology, 325 Broadway, Boulder, Colorado 80305, USA \\ ${ }^{3}$ Institut de Physique Théorique, Université Paris-Saclay, CEA, CNRS, 91191 Gif-sur-Yvette, France
}

(Received 20 May 2019; published 21 August 2019)

\begin{abstract}
Studying a single atomic ion confined in a time-dependent periodic anharmonic potential, we find large amplitude trajectories stable for millions of oscillation periods in the presence of stochastic laser cooling. Accounting for the complexity of the laser cooling process we calculate the details of the effective dynamics away from thermal equilibrium. The competition between energy gain from the time-dependent drive and damping leads to the stabilization of such stochastic limit cycles. Instead of converging to the global minimum of the averaged potential, the steady-state phase-space distribution develops multiple peaks in the regions of phase space where the frequency of the motion is close to a multiple of the periodic drive. Such distinct nonequilibrium behavior can be observed in realistic radio-frequency traps with laser-cooled ions, suggesting that Paul traps offer a well-controlled test bed for studying the transport and dynamics of microscopically driven systems.
\end{abstract}

DOI: 10.1103/PhysRevResearch.1.012012

An atomic ion trapped in near vacuum is a highly isolated system whose quantum motion can be controlled exquisitely [1]. Notwithstanding this, it can also be a system where chaos and randomness at the microscopic level give rise to intriguing classical states of motion.

Paul traps for atomic ions are based on radio-frequency (10-200 MHz) time-dependent potentials [2]. The timedependent drive affects the dynamics qualitatively and the trapping is based on dynamical stabilization, akin to stabilization of an inverted pendulum [3-5]. In general, even for the motion of one particle in one spatial dimension, a time-dependent drive renders the Hamiltonian phase space effectively three dimensional (3D), counting the time (which can be treated as periodic), the space coordinate, and the momentum. For anharmonic potentials, this results in complex phase-space structures.

Laser cooling is widely used in ion trapping [6-16]. With the cooling beam turned on, the ion may be expected to be damped to the minimum of the effective potential or, in some cases [17], heat up or diffuse to a larger amplitude where it may escape from the trap. However, even if the ion is cooled by the laser, the nonequilibrium nature of the dynamics implies in general that the peaks of its spatial probability distribution may not coincide with the minima of the potential. Rather, the distribution may develop new maxima, and complex stochastic limit cycles and hysteretic behavior may emerge [18-20].

\footnotetext{
*haggaila@gmail.com

Published by the American Physical Society under the terms of the Creative Commons Attribution 4.0 International license. Further distribution of this work must maintain attribution to the author(s) and the published article's title, journal citation, and DOI.
}

In this Rapid Communication, we show that the anharmonicity in a periodically driven Paul trap can capture an ion at a large amplitude motion, corresponding to a stable limit cycle with sizable basins of attraction in phase space, even in the presence of damping by laser cooling and the associated randomness. The time dependence of the potential is a critical ingredient for such stochastic limit cycles since, in contrast to time-independent confinement, it prevents the damping from erasing the signatures of the Hamiltonian phase space in the (quasi)stationary state. Instead, the stochastic dynamics retain some of the more complex structure of the underlying Hamiltonian phase space, with multiple peaks of the probability distribution of the ion emerging away from the effective potential minimum. Our approach allows us to calculate the details of the ensuing stochastic dynamics away from thermal equilibrium, accounting for the complex laser cooling process. Although we focus on a trapped ion, the basic required ingredients (time dependence, anharmonicity, and weak damping) are relevant to many dynamically driven systems, as further discussed below.

Model. We consider dynamics in an effectively onedimensional time-periodic potential in the presence of weak damping and first examine the stability of the limit cycles based on an analytic expansion. We then use this expansion to account for the stochastic process of photon scattering from a cooling laser, and further numerically demonstrate that this mechanism remains robust for a realistic trap potential, when the remaining degrees of freedom corresponding to $3 \mathrm{D}$ confinement are taken into account.

In particular, we consider a time-dependent anharmonic potential [21],

$$
V(z, t)=\frac{1}{2} a_{z}\left(z-z_{s}\right)^{2}+q V_{2}(z) \cos 2 t,
$$

where the time $t$ is in units of $2 / \Omega$, with $\Omega$ being the angular frequency of the trap rf drive and the coordinate $z$ is in units of a length scale $d$, defined below. The charge and mass of 


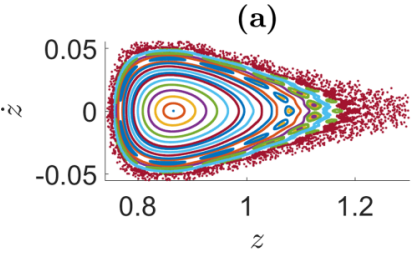

(c)

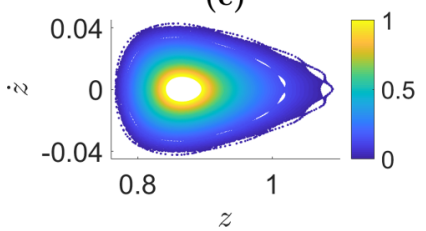

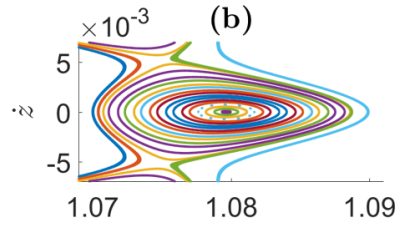

(d)

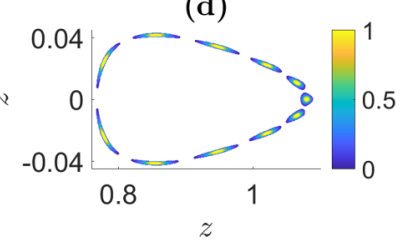

FIG. 1. Stroboscopic maps of the phase space $\{z, \dot{z}\}$ in nondimensional units, obtained numerically by solving the equation of motion for the trap potential $V$ of Eq. (1), and plotting the points the trajectory goes through at times $t=0 \bmod \pi$. (a) Mixed phasespace structure, wherein the chaotic "sea," every closed curve and every "island chain," each one results from one initial condition. See the text for details of the parameters. (b) A closeup of the region around one island of the chain of $s=11$ islands. (c) With deterministic damping added $\left[\gamma=1 \times 10^{-5}\right.$ in Eq. (2)], an ion starting at the rightmost curve in (b) will be slowly damped towards the effective potential minimum (the color code indicates the time along one slowly damping trajectory, where 1 corresponds to $10^{5}$ drive periods). (d) An ion starting deeper within an island of a chain $(\{z=1.087, \dot{z}=0\})$ is captured in a large amplitude motion being damped towards the island centers [same color code as in (c)]. This motion remains stable when generalized to laser-cooling dynamics in three spatial dimensions (see Fig. 2).

the ion and the trap geometry parameters and voltages are absorbed into the nondimensional parameters $q$ and $a_{z}<0$ (the latter resulting from a static harmonic trapping potential along an orthogonal axis, which gives antitrapping along $z$ ). Setting $z_{s}$ to be a point where $V_{2}(z)$ vanishes and choosing the parameters appropriately makes $z_{s}$ a stationary point with stable motion in the phase space around it. Since the periodic drive frequency has been rescaled to $2, V(z, t)$ is $\pi$ periodic.

In numerical simulations we take $V_{2}(z)=$ $\frac{4}{\pi}\left[\arctan \left(\frac{1}{2 z}\right)-\arctan \left(\frac{3}{2 z}\right)\right]$ to be the potential of a model surface trap [21] along an axis perpendicular to the electrode plane (defined by $z=0$, with $d$ the width of two electrodes carrying the rf-modulated voltage). $V_{2}$ vanishes at $z_{s}=\sqrt{3} / 2 \approx 0.866$. The features discussed below can be observed with general anharmonic potentials; expanding $V_{2}$ to order $z^{4}$ about $z_{s}$ is sufficient to obtain the specific limit cycle studied here, although the details would vary. We note, however, that the anharmonic terms are not small, and are not treated here in perturbation theory [22]. We take the values of the dimensionless parameters to be $a_{z}=-0.0008$ and $q \approx 0.87$. This corresponds to realistic trap parameters (see below). For these nondimensional parameters, the analysis of Eq. (1) can be applied to any ion species within such a trap, and any value chosen for $\Omega$ and $d$. Figure 1(a) shows a stroboscopic map of the $3 \mathrm{D}$ phase space, which is obtained by simulating the Hamiltonian dynamics with different initial conditions and stroboscopically plotting $\{z, \dot{z}\}$ at times $t=0 \bmod \pi$.
The phase space is mixed with chaotic and integrable regions. Beyond the central region of closed curves that correspond to regular motion, there is a large chaotic "sea" and some sizable "island chains" of regular motion forming about points where the anharmonic oscillation frequency of the ion is at a rational ratio with the drive frequency. Each closed curve is characterized by the existence of an action that is conserved during the Hamiltonian motion (in contrast, the energy is not conserved, since the potential is time dependent), which is proportional to the enclosed area. Figure 1(b) shows a closeup on the trajectories around one island in a chain which has $s=11$ islands [see Figs. 1(a) and 1(d)]. Due to the chaotic regions, the phase space is fragmented and the action cannot be defined globally, but it is well defined locally on the islands of a chain.

Limit cycles. A trajectory starting at the center of one island of a chain constitutes a periodic orbit repeating itself after $t=$ $s \pi$, with the ion moving between the $s$ island centers in a fixed order. When adding dissipation, the ion is attracted towards the periodic orbit from almost the entire island, a phenomenon previously studied mostly in terms of chaotic maps [23,24]. To model this mechanism of trapping in the island chain, driven solely by damping, we add a friction coefficient $\gamma>0$ to the ion's equation of motion,

$$
\ddot{z}=F(z, t)-\gamma \dot{z}, \quad F(z, t)=-\partial V(z, t) / \partial z .
$$

A numerical simulation of the time evolution using two (different) initial conditions is shown in Figs. 1(c) and 1(d). In Fig. 1(c), the ion starts at $\{z=1.09, \dot{z}=0\}$, too far away from any island center to be attracted and is damped toward $z_{s}$, while in Fig. $1(\mathrm{~d})$ it starts at $\{z=1.087, \dot{z}=0\}$, inside the closed trajectories around one of the island centers and gets trapped in the chain of $s=11$ islands. Such limit cycles are a generic feature that results from the interplay of the nonlinearity of the Hamiltonian forces acting on the ion in the vicinity of island chains, where the time-dependent drive counteracts the damping.

We now consider the dynamics of linearized perturbations about the limit cycle. Assume that $\bar{z}(t)$ is an $s \pi$-periodic orbit that connects the island centers for $\gamma=0$, i.e., $\ddot{\bar{z}}(t)=$ $F[\bar{z}(t), t]$. We write

$$
z(t)=\bar{z}(t)+u(t), \quad \bar{z}=\sum_{n} B_{2 n} e^{i 2 n t / s},
$$

where $n \in \mathbb{Z}$ and $B_{2 n}=B_{-2 n}$ (time-reversal invariance is used in the following). Substituting Eq. (3) into Eq. (2) and linearizing the motion around $\bar{z}(t)$, we get

$$
\ddot{u}+f(t) u=-\gamma(\dot{\bar{z}}(t)+\dot{u}), \quad f(t) \equiv-\frac{\partial F}{\partial z}[\bar{z}(t), t] .
$$

Substituting $u(t)$ by the ansatz $u(t)=w(t) e^{-\gamma t / 2}$ gives

$$
\ddot{w}+\left[f(t)-\frac{\gamma^{2}}{4}\right] w=g(t), \quad g(t) \equiv-\gamma \dot{\bar{z}}(t) e^{\gamma t / 2} .
$$

The general solution for $w$ is composed of the sum of a particular solution growing exponentially as $e^{\gamma t / 2}$, and the two linearly independent solutions of the homogeneous equation [with $g(t)=0$ - see below]. Since $\bar{z}$ is $s \pi$ periodic and $F$ is $\pi$ periodic, the function $f(t)$ in Eq. (4) can be Fourier expanded in the form $f=\sum F_{2 n} e^{i 2 n t / s}$. A particular solution of Eq. (5) 
can be obtained by substituting $w_{0}(t)=e^{\gamma t / 2} \sum W_{2 n} e^{i 2 n t / s}$, which gives an inhomogeneous system of recursion relations for $W_{2 n}$, with a unique solution under general conditions [25]. The homogeneous equation in $w$ [Eq. (5)] with $g(t)=0$, whose coefficients are periodic in time, is a Hill equation [26]. The homogeneous solutions $w(t)$ determine the stability of $u(t)$ [since the exponential growth of the inhomogeneous $w_{0}$ is canceled when going back to $u$ ]. In fact, Eq. (5) with $\gamma=$ $g(t)=0$ determines the linear stability of the periodic orbit in the Hamiltonian case. If the area of the islands about the periodic orbit is not too small, perturbations about the periodic orbit are stable for a range of amplitudes of the motion, which implies that the motion would be linearly stable also for a small nonvanishing value of $\gamma$ in Eq. (5). As Eq. (5) shows, to leading order the damping is eliminated in the dynamics, and indeed we observe numerically that the ion is damped more slowly towards the island centres [Fig. 1(d)], compared to outside the island chain [Fig. 1(c)].

Laser cooling. To incorporate laser cooling more realistically, we assume a beam that is uniform over all positions in $z$ and apply a recently developed semiclassical theory of laser cooling that is valid for motion within the timedependent potential of Paul traps [17]. We approximate the ion as a two-level system, whose excited level has a decay rate (linewidth) $\Gamma$. The validity of our approach requires a low saturation of the internal transition, guaranteed by setting the saturation parameter $s_{\mathrm{L}}$ which is proportional to the laser intensity to $s_{\mathrm{L}} \ll 1$ [17]. A saturation $s_{\mathrm{L}} \sim 0.1$ is often chosen in experiments, since it leads to the lowest final temperatures. The instantaneous Doppler shift due to the ion's velocity determines its probability to absorb a photon at any phase-space point along the trajectory. For optical photons, many scattering events are required in order to change the Hamiltonian action significantly. This allows us to describe the scattering as an adiabatic perturbation leading to a drift and diffusion of the ion between the tori (surfaces of constant action) of the Hamiltonian motion.

With a negative detuning $\Delta \ll-\Gamma$, an ion is efficiently cooled from in the region of high amplitude motion in the approximately integrable part of phase space in a surfaceelectrode trap [17], whereas a laser detuned optimally for reaching the lowest temperatures (with $\Delta \sim-\Gamma / 2$ ) could heat the ion out of the trap from this region. We numerically locate parameters for which a laser beam with $\Delta \ll-\Gamma$ can also capture the ion on trajectories within the island chain in the direct neighborhood of a periodic orbit.

The coordinate $u(t)$ of Eq. (3) represents linearized perturbations (a periodically driven harmonic oscillator) expanded about periodic orbit $\bar{z}(t)$, that can fluctuate, heat up, and be damped by the laser. We numerically calculate $\bar{z}(t), \dot{\bar{z}}(t)$, and $f(t)$ from Eq. (1) (with $\gamma=0$ ), to obtain the Fourier expansion [25] of the Hill oscillator of Eq. (4). This is a key step allowing us to introduce a canonical time-dependent transformation to the Hamiltonian action-angle coordinates $(I, \theta)$ describing the linearized motion about the periodic orbit. By the linearity of the expanded motion, the transformation can be obtained in analytic closed form, with the coefficients of the Fourier expansion calculated numerically. Here, we use the time-reversal invariance of $\bar{z}(t)$, which allows us to employ the theory developed in Refs. [25,27,28] for the
Hill oscillator (accounting for a non-time-reversal invariant drive would require to generalize the theory, which is beyond the scope of the current work). Averaging over the angle $\theta$, we obtain an effective Fokker-Planck equation for the probability distribution $P(I, t)$, which is a probability density function that depends on time and action only [17],

$$
\frac{\partial}{\partial t} P(I, t)=-\frac{\partial}{\partial I} S(I, t) \equiv-\frac{\partial}{\partial I}\left[\Pi_{I} P\right]+\frac{1}{2} \frac{\partial^{2}}{\partial I^{2}}\left[\Pi_{I I} P\right],
$$

with $S(I, t)$ a probability flux, $\Pi_{I}(I)$ an action drift coefficient, and $\Pi_{I I}(I)$ a diffusion coefficient. The calculation of $\Pi_{I}$ and $\Pi_{I I}$ proceeds by using the formulas derived for a linear Floquet system in Ref. [29]. If we find a region of action where the ion remains bounded for a very long time (as determined by the Fokker-Planck dynamics), we can assume an approximately stationary probability distribution in the action, which then takes the form

$$
P(I) \propto\left[\Pi_{I I}(I)\right]^{-1} \exp \left\{2 \int_{0}^{I} d I^{\prime} \Pi_{I}\left(I^{\prime}\right) / \Pi_{I I}\left(I^{\prime}\right)\right\} .
$$

Taking concrete physical parameters we consider a ${ }^{24} \mathrm{Mg}^{+}$ ion. The nondimensional parameters are (see Ref. [21]) $a_{z}=$ $4 e U_{\mathrm{DC}} / m \Omega^{2} c_{z}$ and $q=2 e U_{\mathrm{rf}} / m d^{2} \Omega^{2}$, with $m, e$ the ion's mass and charge, $U_{\mathrm{DC}}, U_{\mathrm{rf}}$ the voltages on the static and rfmodulated electrodes, respectively, $c_{z}$ a geometric constant, and we set $d=150 \mu \mathrm{m}$ and $\Omega=2 \pi \times 20 \mathrm{MHz}$. The resulting oscillation frequency at the effective minimum is $\omega_{z} \approx$ $2 \pi \times 2.3 \mathrm{MHz}$. The laser parameters used in our numerical simulation are $k=\cos (\phi) \times 2 \pi / 280 \mathrm{~nm}^{-1}$ with $\phi \approx 0.4 \pi$ giving the angle between the laser wave vector and the $z$ axis, and $\Gamma \approx 263 \times 10^{6} \mathrm{~s}^{-1}$.

We find a few ranges of a relatively large detuning $-\Delta \gg$ $\Gamma$ for which the periodic orbit is stabilized. For $-11 \Gamma \lesssim \Delta \lesssim$ $-9.8 \Gamma$, we find a stationary distribution in the action within the island chain with a strong (exponential) suppression of the probability of $I$ values away from the island centers. Figure 2(a) presents the action drift coefficient for a few representative values of the detuning, showing that the action drift coefficient is negative throughout most of the island chain, indicating that the ion will drift towards the maximum of the quasistationary action distribution from any close by point on the island. Figure 2(b) shows the corresponding approximately stationary distributions in action. Since this action is expanded about the island centers, and the angles are averaged over by assuming a uniform angle distribution, the corresponding (stroboscopic) phase-space distribution is that depicted schematically in the inset. For $\Delta=-11.2 \Gamma$ we see that the probability density is peaked away from the island center. In this case the exponential suppression of $P(I)$ is weaker than for the other detunings considered here, implying that the lifetime of the limit cycle would be possibly too short to be observed. Due to the stochastic nature of laser cooling, even an ion starting from outside the island chain has a finite probability to diffuse or drift onto one of the islands, however, elucidating this mechanism is beyond the scope of the current work.

Regarding the experimental observability of such a limit cycle, the lifetime within the island can be obtained by calculating the mean first passage time at the island boundary [30], 

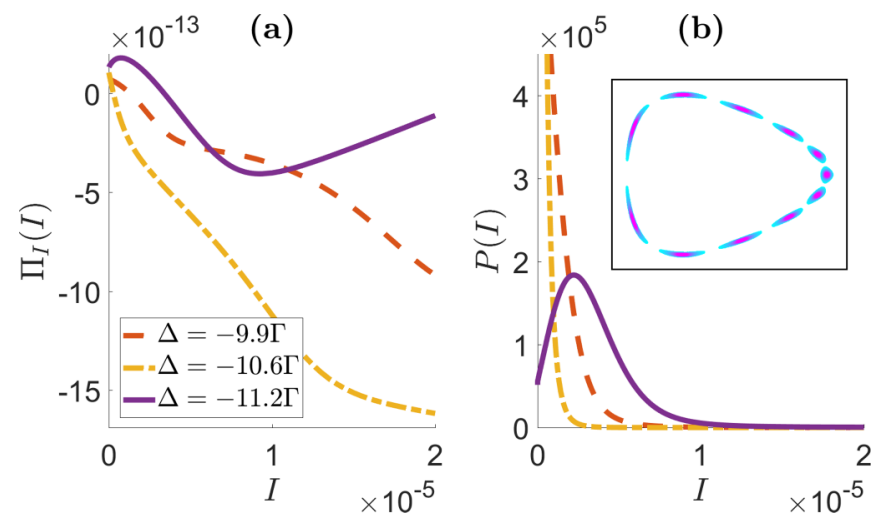

FIG. 2. (a) The action drift coefficient (in nondimensional units - see the text for the parameters) as a function of the action $I$ of linearized motion expanded about the periodic orbit going through the island centers of the chain with $s=11$ islands in Fig. 1(b). Three values of the detuning are shown, with the $I$ axis extending roughly up to the size of (each) island. At action values where the drift coefficient is positive, the ion is effectively heated by scattering laser photons, while it is being cooled where the drift coefficient is negative. (b) The resulting approximately stationary probability distribution [Eq. (7)]. For $-11 \Gamma \lesssim \Delta \lesssim-9.8 \Gamma$ the ion is well cooled within the island chain, with an exponential decay away from the center implying a lifetime estimated to be (for $\Delta=$ $-10.6 \Gamma$ ) of order tens of seconds. The inset shows a schematic depiction of the stroboscopic phase-space distribution (in $z$ and $\dot{z}$, at $t=0 \bmod \pi$ ).

and exceeds tens of seconds around $\Delta=-10.6 \Gamma$ (we also find other ranges of the detuning with shorter lifetimes). The mean photon scattering rate is $1.2 \times 10^{6} \mathrm{~s}^{-1} \times s_{\mathrm{L}}$, with $s_{\mathrm{L}}$ the saturation parameter. This rate can be contrasted with the rate at the effective trap minimum [29], $\left(\Gamma s_{\mathrm{L}} / 2\right) /\left(1+[2 \Delta / \Gamma]^{2}\right)$, which gives $66 \times 10^{6} \mathrm{~s}^{-1} \times s_{\mathrm{L}}$ for the optimal detuning $\Delta=$ $-\Gamma / 2$, but $0.3 \times 10^{6} \mathrm{~s}^{-1} \times s_{\mathrm{L}}$ for $\Delta=-10.6 \Gamma$. The photons can be detected and a Fourier transform of the fluorescence would show peaks at the $s$ subharmonics of the rf drive frequency. We have also simulated the Hamiltonian motion within the full time-dependent potential of the five-wire trap in three spatial dimensions for the parameters analyzed above, verifying that this mechanism is robust with large amplitudes of motion in the remaining coordinates. Island chains frequently develop in surface traps with voltages in the upper range of experimentally relevant values, and they can have very large relative sizes [21]. This is true also for other trap types, since the potential often attains some anharmonic contributions which become relevant above some energy or spatial scale. Hence we expect that such limit cycles can be observed in many existing traps and speculate that they may correspond to metastable extended spatial orbits that have been observed in Paul traps.

To conclude, we have analyzed the dynamics of an ion driven by a periodic potential and stochastically kicked by a velocity-dependent force originating from photon scattering. The balance between the drive, the damping, and the diffusion results in the ion stabilizing in a long-lived limit cycle that corresponds to an extended orbit in space. The probability distribution develops new peaks within the islands of the mixed phase space, centered about a periodic orbit frequency-locked to a rational multiple of the driving frequency. This provides an example of a system driven far out of equilibrium that is nevertheless described by effective dynamics (coarse grained over the angle), which approximately obey a detailed balance [since in the steady state, the probability flux in action, $S(I, t)$ of Eq. (6), must vanish [29], a constraint obeyed by Eq. (7)]. Our calculation manifests a method for obtaining the steadystate distributions for nonequilibrium underdamped systems [31-37] through a mapping of the angle-averaged dynamics to that of an equilibrium system.

The dynamical mechanism we explored is formally close to models of Hamiltonian and Brownian ratchets [38], which are basic models of transport $[39,40]$. Transport in a mixed phase space is especially complex [41-47] and the ability to control and accurately measure motion in complex timedependent potentials make ion-trap experiments suitable for quantitative tests of such ideas [21], extended even to many particles $[48,49]$. Our results can be generalized to frequencylocked limit cycles in the relative coordinate of two interacting particles [50,51], or in the rotation of a macroscopic particle, with $V_{2}$ a periodic function of the rotation angle [52,53].

In the limit of small amplitudes, the coordinate $u(t)$ linearized about the island centers corresponds to a quantum parametric oscillator [25,29]. For the presented parameters, the mean action with $\Delta=-10.6 \Gamma$ corresponds (by using the semiclassical relation $n \approx I / \hbar$, with $n$ the phonon number) to $\langle n\rangle \approx 170$ phonons, somewhat higher than the standard Doppler cooling limit $\langle n\rangle \approx 100$ of an identical ion whose secular frequency is similar, $2 \pi \times 150 \mathrm{kHz}$. Searching for similar limit cycles with larger driving frequencies and different parameters could facilitate cooling this motion to the quantum ground state using the well-developed tools of quantum optics in ion traps (e.g., sideband cooling). This would open up a route for exploring quantum effects in phase space $[46,54,55]$ with a single trapped ion beyond the limit of smallamplitude motion.

Acknowledgments. H.L. thanks Shmuel Fishman, Kirone Mallick, and Roni Geffen for fruitful discussions, and acknowledges support by IRS-IQUPS of Université ParisSaclay and by LabEx PALM under Grant No. ANR-10LABX-0039-PALM.
[1] D. J. Wineland, Nobel lecture: Superposition, entanglement, and raising Schrödinger's cat, Rev. Mod. Phys. 85, 1103 (2013).

[2] W. Paul, Electromagnetic traps for charged and neutral particles, Rev. Mod. Phys. 62, 531 (1990).
[3] P. L. Kapitsa, Collected Papers of P. L. Kapitza (Pergamon, Oxford, UK, 1964).

[4] L. D. Landau and E. M. Lifshitz, Mechanics (ButterworthHeinemann, Oxford, UK, 1976). 
[5] S. Rahav, I. Gilary, and S. Fishman, Effective Hamiltonians for periodically driven systems, Phys. Rev. A 68, 013820 (2003).

[6] D. J. Wineland and W. M. Itano, Laser cooling of atoms, Phys. Rev. A 20, 1521 (1979).

[7] J. Javanainen, Light-pressure cooling of trapped ions in three dimensions, Appl. Phys. 23, 175 (1980).

[8] J. Javanainen and S. Stenholm, Laser cooling of trapped particles I: The heavy particle limit, Appl. Phys. 21, 283 (1980).

[9] J. Javanainen and S. Stenholm, Laser cooling of trapped particles II, Appl. Phys. A: Mater. Sci. Process. 24, 71 (1981).

[10] S. Stenholm, The semiclassical theory of laser cooling, Rev. Mod. Phys. 58, 699 (1986).

[11] J. I. Cirac, L. J. Garay, R. Blatt, A. S. Parkins, and P. Zoller, Laser cooling of trapped ions: The influence of micromotion, Phys. Rev. A 49, 421 (1994).

[12] G. Morigi and J. Eschner, Doppler cooling of a Coulomb crystal, Phys. Rev. A 64, 063407 (2001).

[13] M. Marciante, C. Champenois, A. Calisti, J. PedregosaGutierrez, and M. Knoop, Ion dynamics in a linear radiofrequency trap with a single cooling laser, Phys. Rev. A 82, 033406 (2010).

[14] T. Sikorsky, Z. Meir, N. Akerman, R. Ben-shlomi, and R. Ozeri, Doppler cooling thermometry of a multilevel ion in the presence of micromotion, Phys. Rev. A 96, 012519 (2017).

[15] S. Ejtemaee and P. C. Haljan, 3D Sisyphus Cooling of Trapped Ions, Phys. Rev. Lett. 119, 043001 (2017).

[16] H. Janacek, A. M. Steane, D. M. Lucas, and D. N. Stacey, The effect of atomic response time in the theory of Doppler cooling of trapped ions, J. Mod. Opt. 65, 577 (2018).

[17] A. Maitra, D. Leibfried, D. Ullmo, and H. Landa, Far-fromequilibrium noise-heating and laser-cooling dynamics in radiofrequency Paul traps, Phys. Rev. A 99, 043421 (2019).

[18] T. Pohl, G. Labeyrie, and R. Kaiser, Self-driven nonlinear dynamics in magneto-optical traps, Phys. Rev. A 74, 023409 (2006).

[19] A. E. Kaplan, Single-particle motional oscillator powered by laser, Opt. Express 17, 10035 (2009).

[20] N. Akerman, S. Kotler, Y. Glickman, Y. Dallal, A. Keselman, and R. Ozeri, Single-ion nonlinear mechanical oscillator, Phys. Rev. A 82, 061402(R) (2010).

[21] V. Roberdel, D. Leibfried, D. Ullmo, and H. Landa, Phase-space study of surface-electrode Paul traps: Integrable, chaotic, and mixed motions, Phys. Rev. A 97, 053419 (2018).

[22] G. T. Abraham and A. Chatterjee, Approximate asymptotics for a nonlinear Mathieu equation using harmonic balance based averaging, Nonlinear Dyn. 31, 347 (2003).

[23] U. Feudel, C. Grebogi, B. R. Hunt, and J. A. Yorke, Map with more than 100 coexisting low-period periodic attractors, Phys. Rev. E 54, 71 (1996).

[24] A. J. Lichtenberg and M. A. Lieberman, Regular and Chaotic Dynamics, Applied Mathematical Sciences (Springer, New York, 2010).

[25] H. Landa, M. Drewsen, B. Reznik, and A. Retzker, Classical and quantum modes of coupled Mathieu equations, J. Phys. A: Math. Theor. 45, 455305 (2012).

[26] N. W. McLachlan, Theory and Applications of Mathieu Functions (Clarendon, Oxford, UK, 1947).

[27] H. Landa, M. Drewsen, B. Reznik, and A. Retzker, Modes of oscillation in radiofrequency Paul traps, New J. Phys. 14, 093023 (2012).
[28] H. Kaufmann, S. Ulm, G. Jacob, U. Poschinger, H. Landa, A. Retzker, M. B. Plenio, and F. Schmidt-Kaler, Precise Experimental Investigation of Eigenmodes in a Planar Ion Crystal, Phys. Rev. Lett. 109, 263003 (2012).

[29] H. Landa, Tuning nonthermal distributions to thermal ones in time-dependent Paul traps, Phys. Rev. A 100, 013413 (2019).

[30] N. G. Van Kampen, Stochastic Processes in Physics and Chemistry, 3rd ed. (Elsevier, New York, 2007).

[31] E. Ben-Isaac, É. Fodor, P. Visco, F. van Wijland, and N. S. Gov, Modeling the dynamics of a tracer particle in an elastic active gel, Phys. Rev. E 92, 012716 (2015).

[32] É. Fodor, C. Nardini, M. E. Cates, J. Tailleur, P. Visco, and F. van Wijland, How Far from Equilibrium is Active Matter? Phys. Rev. Lett. 117, 038103 (2016).

[33] V. Dotsenko, A. Maciołek, O. Vasilyev, and G. Oshanin, Two-temperature Langevin dynamics in a parabolic potential, Phys. Rev. E 87, 062130 (2013).

[34] A. Y. Grosberg and J.-F. Joanny, Nonequilibrium statistical mechanics of mixtures of particles in contact with different thermostats, Phys. Rev. E 92, 032118 (2015).

[35] S. N. Weber, C. A. Weber, and E. Frey, Binary Mixtures of Particles with Different Diffusivities Demix, Phys. Rev. Lett. 116, 058301 (2016).

[36] V. Mancois, B. Marcos, P. Viot, and D. Wilkowski, Twotemperature Brownian dynamics of a particle in a confining potential, Phys. Rev. E 97, 052121 (2018).

[37] S. Shankar and M. C. Marchetti, Hidden entropy production and work fluctuations in an active gas, Phys. Rev. E 98, 020604 (2018).

[38] S. Denisov, S. Flach, and P. Hänggi, Tunable transport with broken space-time symmetries, Phys. Rep. 538, 77 (2014).

[39] P. Reimann, Brownian motors: Noisy transport far from equilibrium, Phys. Rep. 361, 57 (2002).

[40] P. Hänggi and F. Marchesoni, Artificial Brownian motors: Controlling transport on the nanoscale, Rev. Mod. Phys. 81, 387 (2009).

[41] H. Schanz, M.-F. Otto, R. Ketzmerick, and T. Dittrich, Classical and Quantum Hamiltonian Ratchets, Phys. Rev. Lett. 87, 070601 (2001).

[42] S. Kraut and U. Feudel, Multistability, noise, and attractor hopping: The crucial role of chaotic saddles, Phys. Rev. E 66, 015207(R) (2002).

[43] S. Denisov, S. Flach, A. A. Ovchinnikov, O. Yevtushenko, and Y. Zolotaryuk, Broken space-time symmetries and mechanisms of rectification of ac fields by nonlinear (non)adiabatic response, Phys. Rev. E 66, 041104 (2002).

[44] R. Ceder and O. Agam, Fluctuations in the relaxation dynamics of mixed chaotic systems, Phys. Rev. E 87, 012918 (2013).

[45] O. Alus, S. Fishman, and J. D. Meiss, Universal exponent for transport in mixed Hamiltonian dynamics, Phys. Rev. E 96, 032204 (2017).

[46] J. Tomkovič, W. Muessel, H. Strobel, S. Löck, P. Schlagheck, R. Ketzmerick, and M. K. Oberthaler, Experimental observation of the Poincaré-Birkhoff scenario in a driven many-body quantum system, Phys. Rev. A 95, 011602(R) (2017)

[47] M. Firmbach, S. Lange, R. Ketzmerick, and A. Bäcker, 3D billiards: Visualization of regular structures and trapping of chaotic trajectories, Phys. Rev. E 98, 022214 (2018). 
[48] J. Brox, P. Kiefer, M. Bujak, T. Schaetz, and H. Landa, Spectroscopy and Directed Transport of Topological Solitons in Crystals of Trapped Ions, Phys. Rev. Lett. 119, 153602 (2017).

[49] B. Liebchen and P. Schmelcher, Interaction induced directed transport in ac-driven periodic potentials, New J. Phys. 17, 083011 (2015).

[50] J. Hoffnagle and R. G. Brewer, Frequency-Locked Motion of Two Particles in a Paul Trap, Phys. Rev. Lett. 71, 1828 (1993).

[51] J. Hoffnagle and R. G. Brewer, On the frequency-locked orbits of two particles in a Paul trap, Science 265, 213 (1994).
[52] B. E. Kane, Levitated spinning graphene flakes in an electric quadrupole ion trap, Phys. Rev. B 82, 115441 (2010).

[53] T. Delord, L. Nicolas, Y. Chassagneux, and G. Hétet, Strong coupling between a single nitrogen-vacancy spin and the rotational mode of diamonds levitating in an ion trap, Phys. Rev. A 96, 063810 (2017).

[54] O. Bohigas, S. Tomsovic, and D. Ullmo, Manifestations of classical phase space structures in quantum mechanics, Phys. Rep. 223, 43 (1993).

[55] C. Grossert, M. Leder, S. Denisov, P. Hänggi, and M. Weitz, Experimental control of transport resonances in a coherent quantum rocking ratchet, Nat. Commun. 7, 10440 (2016). 